\title{
Response Evaluation Criteria in Solid Tumors
}

National Cancer Institute

\section{Source}

National Cancer Institute. Response Evaluation Criteria in Solid Tumors. NCI Thesaurus.

Code C49164.

Standard parameters to be used when documenting response of solid tumors to treatment; a set of published rules that define when cancer patients improve ("respond"), stay the same ("stable"), or worsen ("progression") during treatments. (from www.recist.com) 